\title{
Maximal and submaximal oxygen uptake efficiency slope: influence of cardiorespiratory variables and maximal dynamic strength
}

\author{
Leonardo A Pasqua ${ }^{1,4 *}$, Mayara V Damasceno ${ }^{1,2}$, Adriano E Lima-Silva ${ }^{1,3}$ and Romulo Bertuzzi $^{3}$ \\ ${ }^{1}$ Endurance Performance Research Group, School of Physical Education and Sport, University of São Paulo. Av. Prof. Melo Moraes, 65, Cidade Universitária, SP, Brazil \\ ${ }^{2}$ Physical Education Department, CESMAC University Center, Brazil \\ ${ }^{3}$ Human Performance Research Group, Academic Department of Physical Education (DAEFI), Technological Federal University of Parana, Curitiba, PR, Brazil \\ ${ }^{4}$ Physical Education, Federal University of Alagoas, AL, Brazil
}

\begin{abstract}
Objective: The purpose of this study was to investigate if the OUES is determined by the same factors when calculated with $\mathrm{VO}_{2}$ and $\mathrm{VE}$ values throughout the incremental test $\left(\mathrm{OUES}_{100}\right)$ or using values until $80 \%$ of the $\mathrm{VO}_{2} \max \left(\mathrm{OUES}_{80}\right)$.

Methods: 116 healthy male individuals performed a maximal incremental test, two constant-speed tests and a maximal dynamic strength test.

Results: OUES ${ }_{100}$ and OUES ${ }_{80}$ were significantly correlated $(\mathrm{r}=0.964 ; P<0.001)$. $\mathrm{VO}_{2}$ max was the main determinant factor for both OUES ${ }_{100}$ and OUES ${ }_{80}$ Additionally, maximal heart rate (HRmax) and maximal dynamic strength (1RM) were the other variables selected for the two models as secondary variables. However, the importances of them were inversed, with the HRmax being the second determinant of the OUES ${ }_{80}$, while 1 RM the second determinant for OUES ${ }_{100}$. The running economy, ventilatory threshold and respiratory compensation point were not selected for the models $(P>0.05)$.

Conclusions: Our results suggest that the $\mathrm{OUES}_{80}$ might satisfactorily replace maximal variables in aerobic fitness evaluations without maximal effort requirement. $\mathrm{VO}_{2}$ max seems to be the main factor determining oxygen uptake efficiency slope, regardless the range (maximal or submaximal) used to determine. Otherwise, the muscle strength plays a more important role to the oxygen uptake efficiency considering maximal intensities, while the cardiovascular system seems to more strongly influence the oxygen uptake efficiency only until submaximal intensities.
\end{abstract}

\section{Introduction}

Aerobic fitness has been considered an important marker for both health and athletic performance in different populations [1]. Traditionally, the aerobic fitness has been determined by some cardiorespiratory variables such as the maximal oxygen uptake $\left(\mathrm{VO}_{2} \max \right)$ [2]. The $\mathrm{VO}_{2} \max$ represents the highest rate of uptake, transport, and consumption of the atmospheric oxygen during exercise [3]. Despite its large utility, $\mathrm{VO}_{2}$ max determination demands the attainment of maximal effort, what in turn is not always possible for all individuals [4]. In addition, the maximal effort could trigger uncomfortable effects in some special populations, such as chronic heart failure patients [5]. Hence, several submaximal aerobic fitness markers have been proposed in the literature to overlap this limitation.

Among the main submaximal cardiorespiratory parameters, the Ventilatory Threshold (VT), the Respiratory Compensation Point (RCP) and the Running Economy (RE) are underlined. VT and RCP are related to changes in blood $\mathrm{H}^{+}$concentrations and represent physiological transition points demarking different exercise intensity domains [6]. In turn, $\mathrm{RE}$ is defined as the oxygen consumption demanded for a given running speed [7]. While these cardiorespiratory parameters are closely associated with aerobic fitness, the detection of the VT and the RCP may be biased by several factors including the different methods of detection and the experience of the evaluators [8-10]. On the other hand, the RE measurement demands at least an additional experimental session, mainly when two or more speeds will be tested or when the individual has a low fitness level. Unfortunately, this time-consuming procedure might discourage exercise physiologists for using RE in their evaluation routines. Therefore, lesser timedemanding methods to assess aerobic fitness would be very appealing from an applied standpoint.

Regarding these concerns, Baba et al. [11] proposed the Oxygen Uptake Efficiency Slope (OUES) as a parameter representing the oxygen uptake efficiency. The OUES has been evaluated during incremental exercise tests, which would demand a unique test session. The OUES is determined by the slope of the linear relationship between oxygen consumption $\left(\mathrm{VO}_{2}\right)$ and the logarithmic transformation of the Ventilation (VE)values during an incremental exercise test. Its determination can be made using total (i.e., 100\%) or partial (e.g., $80 \%)$ gas exchange data collected during the incremental test $[12,13]$.

Correspondence to: Leonardo Alves Pasqua, Study Group on Aerobic Performance (GEDAE-USP) - School of Physical Education and Sport, University of São Paulo. Av. Prof. Mello Moraes, 65 - Butantã, São Paulo, Brazil, Tel. 5511 98775-5719; Fax. 5511 3813-5091, Email: leonardopasqua@gmail.com

Key words: aerobic fitness, maximal oxygen uptake, maximal heart rate, muscle strength, oxygen uptake efficiency, OUES

Received: July 20, 2017; Accepted: August 17, 2017; Published: August 21, 2017 
It is believed that the OUES integrates in a unique physiological index respiratory (e.g., oxygen perfusion in the lungs and pulmonary dead space), cardiovascular (e.g., blood flow distribution to the exercised muscles), and muscular (e.g., oxygen extraction and utilization) functions [14]. In fact, it has been used to evaluate aerobic fitness in a wide number of subjects, including healthy men [12], overweight adolescents [15], and cardiovascular disease patients [12]. It is interesting to note that the OUES determination is not influenced by some intervenient factors, such as the use of experienced and nonexperienced evaluators or test protocol [12]. Moreover, the OUES measurement can be performed without necessarily require a maximal effort or additional experimental sessions [16].

Although it has been well accepted that aerobic fitness is highly dependent on cardiorespiratory system [17], several studies have also found a strong association between muscle strength and aerobic fitness $[18,19]$. Individuals with greater muscle strength could generate lower relative force at the same absolute running intensity. This lower relative force might result in an increased local blood flow, augmenting the oxygen extraction and utilization [20]. As a result, this increased oxygen availability to the working muscles could improve muscular metabolism and control acidosis in the muscle fibers [18]. In fact, a $12 \%$ improvement in healthy men $\mathrm{VO}_{2}$ max was found after a 12 -week strength-training [21]. On this basis, the muscle strength seems to influence important processes and may be important to OUES determination. However, to the best of our knowledge, the possible relationship between maximal dynamic muscle strength and OUES during running was not still analyzed.

Therefore, the objective of the current study was to investigate the main determinants of OUES calculated with maximal (i.e., 100\%) and submaximal (i.e., 80\%) intensities during running in healthy individuals. It was hypothesized that OUES would be associated with $\mathrm{VO}_{2}$ max, ventilatory thresholds, $\mathrm{RE}$, and maximum dynamic strength. In case of confirming this association, it could be argued that OUES might be used as a general aerobic fitness marker summarizing different physiological and muscular parameters.

\section{Methods}

116 healthy male individuals participated in this study after the assignment of a written informed consent. All experimental procedures were previously approved by the Ethics Committee for Humans Studies from the School of Physical Education and Sport of University of São Paulo (2010/44). Participants were asked to refrain from any exhaustive or unaccustomed exercise in the $48 \mathrm{H}$ preceding each test session, and from taking nutritional supplements throughout the experimental period.

A maximal incremental test was performed to determine the $\mathrm{VO}_{2}$ max, maximal heart rate (HRmax), VT and RCP. Specific details of the maximal incremental test, as well as the physiological variables determination, have been described elsewhere [22].

The two constant-speed tests were performed to determine the running economy at $10 \mathrm{~km} \cdot \mathrm{h}^{-1}\left(\mathrm{RE}_{10}\right)$ and $12 \mathrm{~km} \cdot \mathrm{h}^{-1}\left(\mathrm{RE}_{12}\right)$. Specific details of the constant speed tests and RE determination have been described elsewhere ${ }^{22}$.

Participants were familiarized with all procedures, leg press machine equipment, and proper exercise technique prior to maximal dynamic strength test. After a brief warm up of five minutes run at 8 $\mathrm{kmh}^{-1}$, participants performed two sets, being five repetitions at $50 \%$ of 1RM determined in the familiarization session for the first set, and three repetitions at $70 \%$ for the second set. After that, participants rested for three minutes. Then, they had up to five trials to achieve the $1 \mathrm{RM}$ load (maximum weight lifted once with the proper technique), with a 3-minute interval between attempts.

The relationship between $\mathrm{VO}_{2}$ and $\mathrm{VE}$ during the incremental test is best described by a single exponential function. Thus, the OUES was determined from $\mathrm{VO}_{2}$ and $\mathrm{VE}$ data during the incremental running test using the equation 1 , as suggested by Baba et al. ${ }^{11}$ :

$$
\mathrm{VO}_{2}\left(\mathrm{~L} \cdot \mathrm{min}^{-1}\right)=\mathrm{a} \cdot \log _{10} \mathrm{~V} \mathrm{E}\left(\mathrm{L} \cdot \mathrm{min}^{-1}\right)+\mathrm{b}
$$

Excluding the warm-up period, $\mathrm{VO}_{2}\left(\mathrm{~mL} \cdot \mathrm{min}^{-1}\right)$ during the incremental test was plotted on the $y$ axis and the VE (L.min ${ }^{-1}$ ) on the $x$ axis after a semilog transformation. The slope of this relation, determined by the angular coefficient "a", represents the OUES. The constant " $b$ " is the linear coefficient of the relation. The OUES was calculated using $100 \%$ of the data $\left(\mathrm{OUES}_{100}\right)$ and with the data contained until $80 \%$ of the $\mathrm{VO}_{2} \max \left(\mathrm{OUES}_{80}\right)$.

Data normality in all the variables was confirmed through Kolmogorov-Smirnov test. Data are expressed by means \pm standard deviations, and 95\% confidence intervals (CI). Pearson productmoment coefficient was used to test the correlation between OUES and $\mathrm{OUES}_{80}$. Two stepwise multiple linear regression models were used to identify which variable(s) explained the OUES $_{100}$ and the OUES variance. The parameters $\mathrm{VO}_{2} \max , \mathrm{HRmax}, \mathrm{VT}, \mathrm{RCP}, \mathrm{RE} 10, \mathrm{RE} 12$ and $1 R M$ were considered as independent variables, whereas OUES ${ }_{100}$ and OUES $_{80}$ were considered as dependent variables in each model. All the statistical analyses were conducted using the SPSS statistical package (version 16.0, Chicago, USA). The significance level was set at $\alpha=0.05$ for all statistical analysis.

\section{Results}

Table 1 shows the main characteristics of the participants. The mean $( \pm \mathrm{SD})$, and $95 \%$ CI OUES ${ }_{100}, \mathrm{OUES}_{80}$, cardiorespiratory parameters, and muscle strength are presented in table 2 (Table 1 and Table 2).

OUES $_{100}$ and OUES 80 were significantly correlated $(r=0.964 ; P<$ 0.001) (Figure 1). The stepwise multiple regression with the OUES as the depen $\mathrm{V}_{2}$ max le resulted in a final model with (in order of importance) $\mathrm{VO}_{2} \max , 1 \mathrm{RM}$, and HRmax as predictors of OUES $\mathrm{O}_{100^{\circ}}$ Otherwise, VT $(P=0.620), \operatorname{RCP}(P=0.553)$, RE10 $(P=0.112)$, and RE12 $(P=0.134)$ were not included in the model. The stepwise multiple regression with the $\mathrm{OUES}_{80}$ as the dependent variable resulted in a final model with (in order of importance) $\mathrm{VO}_{2}$ max, HRmax, and $1 \mathrm{RM}$ as

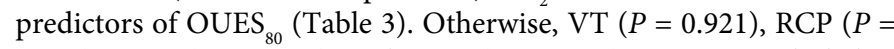
$0.562)$, RE10 $(P=0.074)$, and RE12 $(P=0.085)$ were not included in the model.

\section{Discussion}

The present study was conducted aiming to verify if submaximal OUES might represent maximal values as well as to identify the main

Table 1. Age and anthropometric characteristics of the participants $(n=116)$.

\begin{tabular}{|c|c|c|}
\hline $\begin{array}{c}\text { Characteristics of the } \\
\text { participants }\end{array}$ & Mean \pm SD & 95\% Confidence interval \\
\hline Age (years) & $25.9 \pm 3.9$ & $24.8-26.6$ \\
\hline Body mass $(\mathrm{kg})$ & $78.9 \pm 14.1$ & $74.8-79.4$ \\
\hline Height $(\mathrm{cm})$ & $176 \pm 13$ & $176-178$ \\
\hline Body fat $(\%)$ & $13.5 \pm 4.1$ & $12.8-14.8$ \\
\hline BMI $\left(\mathrm{kg} . \mathrm{m}^{-2}\right)$ & $25.2 \pm 4.8$ & $24.3-26.2$ \\
\hline
\end{tabular}

BMI: Body Mass Index 


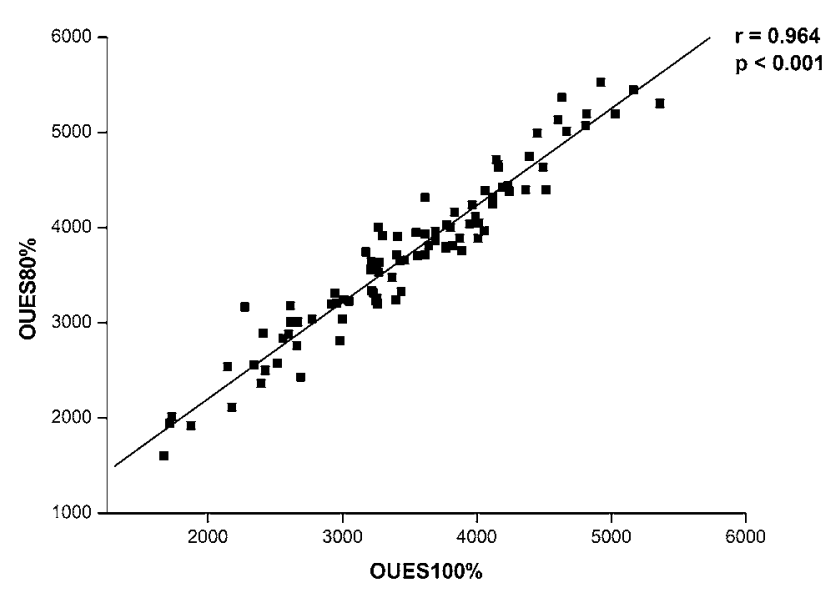

Figure 1. Correlation between oxygen uptake efficiency slope calculated using $80 \%$ $\left(\mathrm{OUES}_{80}\right)$ and $100 \%\left(\mathrm{OUES}_{100}\right)$ of the gas exchange data collected during a maximal incremental test $(\mathrm{n}=116)$.

Table 2. Oxygen uptake efficiency slope, cardiorespiratory parameters and muscle strength $(\mathrm{n}=116)$

\begin{tabular}{|c|c|c|}
\hline & Mean \pm SD & 95\% Confidence interval \\
\hline OUES $_{100}$ & $3442 \pm 611$ & $3291-3564$ \\
\hline OUES $_{80}$ & $3738 \pm 885$ & $3555-3920$ \\
\hline VO $_{2} \max \left(\mathrm{mL} \cdot \mathrm{kg}^{-1} \cdot \mathrm{min}^{-1}\right)$ & $48.7 \pm 6.0$ & $46.3-49.2$ \\
\hline $\mathrm{HRmax}(\mathrm{bpm})$ & $189 \pm 9$ & $187-192$ \\
\hline $\mathrm{VT}\left(\mathrm{km} \cdot \mathrm{h}^{-1}\right)$ & $10.5 \pm 1.0$ & $10.3-10.8$ \\
\hline $\mathrm{RCP}\left(\mathrm{km} \cdot \mathrm{h}^{-1}\right)$ & $13.5 \pm 1.5$ & $13.2-13.9$ \\
\hline $\mathrm{RE} 10\left(\mathrm{~mL} \cdot \mathrm{kg}^{-1} \cdot \mathrm{min}^{-1}\right)$ & $36.6 \pm 3.2$ & $36.0-37.4$ \\
\hline $\mathrm{RE12}\left(\mathrm{mL} \cdot \mathrm{kg}^{-1} \cdot \mathrm{min}^{-1}\right)$ & $41.9 \pm 3.6$ & $41.4-43.0$ \\
\hline $1 \mathrm{RM}(\mathrm{kg})$ & $305 \pm 73$ & $285-319$ \\
\hline
\end{tabular}

$\mathrm{V}_{2}$ max: Maximal oxygen uptake; HRmax: Maximal heart rate; VT: Ventilatory threshold; RCP: Respiratory Compensation Point; OUES ${ }_{100}$ : Oxygen Uptake Efficiency slope measured with $100 \%$ of the incremental test data; OUES : Oxygen Uptake Efficiency slope measured with data until $80 \%$ of the incremental test; RE10: Running Economy measured at $10 \mathrm{~km} \cdot \mathrm{h}^{-1}$; RE12: Running Economy measured at $12 \mathrm{~km} \cdot \mathrm{h}^{-1}$; 1RM: one Repetition Maximum in the leg press exercise.

Table 3. Predictors of the oxygen uptake efficiency slope at $100 \%$ and $80 \%$. $(n=116)$

\begin{tabular}{|c|c|c|c|c|c|}
\hline \multirow{2}{*}{$\begin{array}{c}\text { Dependent } \\
\text { variable }\end{array}$} & $\begin{array}{c}\text { Independent } \\
\text { Variables }\end{array}$ & $\begin{array}{c}\text { Standardized } \\
\text { coefficient }\end{array}$ & Partial $\mathrm{r}$ & $\mathrm{p}$ value & \multirow{2}{*}{ Adjusted $\mathrm{r}^{2}$} \\
\hline \multirow{3}{*}{ OUES $_{100}$} & $\mathrm{VO}_{2} \mathrm{max}$ & 0.621 & 0.614 & 0.001 & \multirow{2}{*}{0.405} \\
\cline { 2 - 5 } & $1 \mathrm{RM}$ & 0.296 & 0.349 & 0.001 & \\
\cline { 2 - 6 } & $\mathrm{HRmax}$ & -0.192 & -0.239 & 0.031 & \\
\hline \multirow{3}{*}{ OUES $_{80}$} & $\mathrm{VO}_{2} \max$ & 0.605 & 0.602 & 0.001 & \multirow{2}{*}{0.379} \\
\cline { 2 - 6 } & $\mathrm{HRmax}$ & -0.303 & -0.362 & 0.001 & \\
\cline { 2 - 6 } & $1 \mathrm{RM}$ & 0.193 & 0.235 & 0.04 & \\
\hline
\end{tabular}

OUES $_{100}$ : oxygen uptake efficiency slope measured with $100 \%$ of the incremental test data; $\operatorname{OUES}_{80}$ : oxygen uptake efficiency slope measured with data until $80 \%$ of the incremental test; $\mathrm{vO}_{2} \max =$ maximal oxygen uptake; $1 \mathrm{RM}=$ repetition maximum in leg press exercise; HR: maximal heart rate.

factors determining OUES calculated from maximal and submaximal gas exchange data range. For this purpose, we used a stepwise multiple regression model to evaluate the contribution of cardiorespiratory and muscular parameters for OUES ${ }_{100}$ and OUES $_{80}$ determination during running in healthy individuals. Our main results were that maximal and submaximal OUES were significantly correlated and both were mainly determined by the $\mathrm{VO}_{2}$ max. Additionally, HRmax and $1 \mathrm{RM}$ were the other selected variables in the two models, but in an inverse order.

Our data demonstrated that OUES $_{100}$ and OUES $_{80}$ were positively correlated. Similarly, previous studies demonstrated that maximal and submaximal OUES were positively correlated. Van Laethem et al. [23] observed a significant correlation between OUES measured until $90 \%$ of the incremental test and OUES with maximal data $(\mathrm{r}=$ 0.973; $\mathrm{p}<0.0001)$ in 35 male and female patients with chronic heart failure. Williamson et al. [24] also observed that full test OUES was significantly correlated with OUES until RER 1.0 was reached $(r=$ $0.90)$ and with OUES until RER 0.9 was reached $(r=0.79)(p<0.05)$ in 100 healthy men. These results support a linear relationship between maximal and submaximal OUES and suggest that the submaximal OUES might be an interesting parameter to replace maximal OUES in individuals whom are unable to reach maximal effort.

Interestingly, data from the multiple regression models selected only variables measured during maximal effort to explain the variation of both OUES ${ }_{100}$ and $\mathrm{OUES}_{80}$ (i.e., $\mathrm{VO}_{2}$ max HRmax, and 1RM). This is in line with previous findings [12], reinforcing the capacity of the OUES measured at submaximal intensities to reflect maximal parameters. Furthermore, $\mathrm{VO}_{2}$ max was the main determinant explaining 60\% of the OUES $_{100}$ and OUES ${ }_{80}$ variance. The positive relationship between $\mathrm{VO}_{2}$ max and OUES found in this study has been found in other studies.

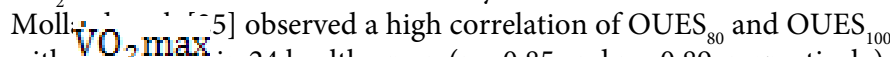
with $\mathrm{O}_{2} \mathrm{max}_{\text {in }} 24$ healthy men $(\mathrm{r}=0.85$ and $\mathrm{r}=0.89$, respectively). In addition, Baba et al. [26] observed a significant correlation $(r=0.78)$ between OUES and $\mathrm{VO}_{2}$ peak. Considering that $\mathrm{VO}_{2}$ max is influenced by both maximal cardiac output and a-v $\mathrm{O}_{2}$ difference, it could be proposed that the OUES is linked to these cardiorespiratory factors and would represent a parameter of aerobic fitness, but with the advantage to be determined using submaximal intensities. It seems especially important, particularly in special populations such as cardiovascular disease patients, which might be unable to complete a test until to exhaustion [12].

It is interesting to observe that the both $1 \mathrm{RM}$ and HRmax were selected by multiple regression models to explain the partial variances of the OUES ${ }_{100}$ and OUES ${ }_{80}$ (Tables 3 and 4), but in a different order of importance. While 1RM was the second more important parameter for the OUES ${ }_{100}$, HRmax was selected as the second more important factor explaining the partial variances for the $\mathrm{OUES}_{80}$. This suggests that, although muscle tissue and cardiovascular system were relevant to both OUES ${ }_{100}$ and OUES ${ }_{80}$, maximal dynamic strength might be more important to OUES ${ }_{100}$, while cardiovascular function might be more important to $\mathrm{OUES}_{80}{ }^{100}$. It is plausible to assume that the muscle strength is critical to the oxygen uptake efficiency in the last stages of the incremental test because there is a higher demand of force production at higher running intensities [27]. On the other hand, the cardiac work might be determinant to the oxygen uptake efficiency at submaximal speeds, since heart rate is an index of the rate-pressure product, a marker of cardiac work [28]. As suggested by our results, OUES $_{80}$ is negatively related to HRmax, what suggests that the submaximal oxygen uptake efficiency increases as the cardiac work decreases at submaximal intensities. Therefore, these findings suggest that the oxygen uptake efficiency could be differently determined accordingly to the intensity of measurement. From the practical standpoint, it seems especially important that exercise physiologists consider that, depending on the intensity of the OUES determination, it might be more dependent on cardiovascular or muscular functions, what should be fit with the evaluated subject or population.

As aforementioned, the stepwise regression models selected the HRmax as a determinant of both OUES $_{100}$ and OUES $_{80}$. It is known that the aerobic fitness level is associated with low values of HRmax in healthy individuals, what has been related to high values of maximal 
cardiac output [29], high stroke volume and hypertrophy of the left ventricle [30]. This is in agreement with the findings of Fu et al. [31], whom observed higher values of cardiac output after 12 weeks of aerobic training accompanied by an improvement in the OUES. Thereby, it is tempter to suggest that the oxygen uptake efficiency may be increased by similar mechanisms responsible to decrease HRmax such as cardiac output enhancement [29] and parasympathetic nervous system activity [32]. Thus, future studies should investigate the relationship between the parameters able to regulate HRmax (e.g., maximal cardiac output, autonomic nervous system, and stroke volume) and OUES.

Our multiple regression models also selected $1 \mathrm{RM}$ as a determinant of the OUES ${ }_{100}$ and the OUES ${ }_{80}$. Once the muscle strength is highly determined by muscle mass, this result confirms the previous suggestion of Baba et al. [11] and Akkerman et al. [33] that the OUES depends, among other factors, on the muscle mass able to extract oxygen from the blood and utilize it in bioenergetic processes. Furthermore, during running, a higher muscle strength in the lower limbs is able to provide a lower time of occlusion and a higher blood flow to the exercised muscles [18,20]. For instance, Storen et al. [18] observed that the improvements in $1 \mathrm{RM}$ after 8 weeks of strength training were accompanied by a significant increase in time to exhaustion at maximal aerobic speed. In this sense, OUES may be related to muscle strength through the improvement of blood flow to the exercise muscles during running. This increased blood flow could positively influence the oxygen uptake by augmenting the oxygen transit in muscle capillaries per time unit during exercise in stronger individuals. This increased oxygen transit would be important for oxygen and substrates offer to the working muscles, and to metabolites clearance, both of which would improve bioenergetic processes and the control of the acidosis in the muscle fibers [18].

The present study has some limitations. Mainly, we used only healthy subjects, in spite of the highlighted advantage of the OUES to special populations [26]. However, our results might be used as standard parameters of a normal population of healthy young men, similarly with the results of Buiys et al. [34], in order to compare with participants with other characteristics, such as cardiovascular disease patients. Moreover, once we used treadmill running and the type of exercise influences physiological variables and their associations with OUES [35], the data should be seen with caution and interpreted in an ergometer-manner dependent way.

\section{Conclusion}

In conclusion, the results of the present study demonstrate that both maximal and submaximal OUES are determined by the maximal oxygen uptake, muscle strength and maximal heart rate (i.e., $\mathrm{VO}_{2} \max$ , 1RM, and HRmax, respectively). While the $\mathrm{VO}_{2}$ max was the main determinant of the OUES regardless the intensity range used, 1RM and HRmax were more important for OUES ${ }_{100}$ and OUES ${ }_{80}$, respectively. Thus, it seems that at maximal intensities the muscle strength plays a more important role to the oxygen uptake efficiency. Otherwise, at submaximal intensities, the cardiovascular system seems to exert a strongly influence on the oxygen uptake efficiency. Despite the submaximal OUES is able to reflect maximal values, it is important to consider the determinant factors of oxygen uptake efficiency in different intensity ranges.

\section{Acknowledgements}

The authors would like to thank São Paulo Research Foundation by the financial support as master and doctorate scholarships (FAPESP: 2010/13913-6; FAPESP: 2013/00371-9).

\section{References}

1. Armstrong N, Tomkinson G, Ekelund U (2011) Aerobic fitness and its relationship to sport, exercise training and habitual physical activity during youth. Br J Sports Med 45: 849-858. [Crossref]

2. Saltin B, Astrand PO (1967) Maximal oxygen uptake in athletes. J Appl Physiol 23 353-358. [Crossref]

3. Bassett DR Jr, Howley ET (2000) Limiting factors for maximum oxygen uptake and determinants of endurance performance. Med Sci Sports Exerc 32: 70-84. [Crossref]

4. Ingle L, Witte KK, Cleland JG, Clark AL (2008) The prognostic value of cardiopulmonary exercise testing with a peak respiratory exchange ratio of $<1.0$ in patients with chronic heart failure. Int J Cardiol 127: 88-92. [Crossref]

5. Antoine-Jonville S, Pichon A, Vazir A, Polkey MI, Dayer MJ (2012) Oxygen uptake efficiency slope, aerobic fitness, and V(E)-VCO2 slope in heart failure. Med Sci Sports Exerc 44: 428-434. [Crossref]

6. Ozyener F, Rossiter HB, Ward SA, Whipp BJ (2001) Influence of exercise intensity on the on- and off-transient kinetics of pulmonary oxygen uptake in humans. J Physiol 533: 891-902. [Crossref]

7. Saunders PU, Pyne DB, Telford RD, Hawley JA (2004) Factors affecting running economy in trained distance runners. Sports Med 34: 465-485. [Crossref]

8. Yeh MP, Gardner RM, Adams TD, Yanowitz FG, Crapo RO (1983) "Anaerobic threshold": problems of determination and validation. J Appl Physiol Respir Environ Exerc Physiol 55: 1178-1186. [Crossref]

9. Gladden LB, Yates JW, Stremel RW, Stamford BA (1985) Gas exchange and lactate anaerobic thresholds: inter- and intraevaluator agreement. J Appl Physiol (1985) 58: 2082-2089. [Crossref]

10. Shimizu M, Myers J, Buchanan N, Walsh D, Kraemer M, et al. (1991) The ventilatory threshold: method, protocol, and evaluator agreement. Am Heart J 122: 509-516. [Crossref]

11. Baba R, Nagashima M, Goto M, Nagano Y, Yokota M, et al. (1996) Oxygen uptake efficiency derived from the relation between oxygen uptake and minute ventilation during incremental exercise. J Am Coll Cardiol 28: 1567-1572. [Crossref]

12. Hollenberg M and Tager IB (2000) Oxygen uptake efficiency slope: an index of exercise performance and cardiopulmonary reserve requiring only submaximal exercise. $J \mathrm{Am}$ Coll Cardiol 36: 194-201. [Crossref]

13. Mourot L, Perrey S, Tordi N, Rouillon JD (2004) Evaluation of fitness level by the oxygen uptake efficiency slope after a short-term intermittent endurance training. Int $J$ Sports Med 25: 85-91. [Crossref]

14. Mezzani A, Agostoni P, Cohen-Solal A, Corrà U, Jegier A, et al. (2009) Standards for the use of cardiopulmonary exercise testing for the functional evaluation of cardiac patients: a report from the Exercise Physiology Section of the European Association for Cardiovascular Prevention and Rehabilitation. Eur J Cardiovasc Prev Rehabil 16:249267. [Crossref]

15. Drinkard B, Roberts MD, Ranzenhofer LM, Han JC, Yanoff LB, et al. (2007) Oxygenuptake efficiency slope as a determinant of fitness in overweight adolescents. Med Sci Sports Exerc 39: 1811-1816. [Crossref]

16. Pogliaghi S, Dussin E, Tarperi C, Cevese A, Schena F (2007) Calculation of oxygen uptake efficiency slope based on heart rate reserve end-points in healthy elderly subjects. Eur J Appl Physiol 101: 691-696. [Crossref]

17. Blaber AP, Walsh ML, Carter JB, Seedhouse EL, Walker VE (2004) Cardiopulmonary physiology and responses of ultramarathon athletes to prolonged exercise. Can J Appl Physiol 29: 544-563. [Crossref]

18. Støren O, Helgerud J, Støa EM, Hoff J (2008) Maximal strength training improves running economy in distance runners. Med Sci Sports Exerc 40: 1087-1092. [Crossref]

19. Bertuzzi R, Lima-Silva AE, Pires FO, et al. (2013) Pacing strategy determinants during a 10-km running time trial: contributions of perceived effort, physiological, and muscular parameters. J Strength Cond Res 28: 1688-1696. [Crossref]

20. Hoff J, Gran A, Helgerud J (2002) Maximal strength training improves aerobic endurance performance. Scand J Med Sci Sports 12: 288-295. [Crossref]

21. Gettman LR, Ward P, Hagan RD (1982) A comparison of combined running and weigh training with circuit weight training. Med Sci Sports Exerc 14: 229-234. [Crossref] 
22. Pasqua LA, Bueno S, Matsuda M, Marquezini MV, Lima-Silva AE, et al. (2016) The genetics of human running: ACTN3 polymorphism as an evolutionary tool improving the energy economy during locomotion. Ann Hum Biol 43: 255-260. [Crossref]

23. Van Laethem C, Van De Veire N, De Backer G, Bihija S, Seghers T, et al. (2007) Response of the oxygen uptake efficiency slope to exercise training in patients with chronic heart failure. Eur J Heart Fail 9: 625-629. [Crossref]

24. Williamson W, Fuld J, Westgate K (2012) Validity of reporting oxygen uptake efficiency slope from submaximal exercise using respiratory exchange ratio as secondary criterion. Pulm Med 874020.

25. Mollard P, Woorons X, Antoine-Joinville S, Jutand L, Richalet JP, et al. (2008) 'Oxygen uptake efficiency slope' in trained and untrained subjects exposed to hypoxia. Respir Physiol Neurobiol 161: 167-173. [Crossref]

26. Baba R, Kubo N, Morotome Y, Iwagaki S (1999) Reproducibility of the oxygen uptake efficiency slope in normal healthy subjects. J Sports Med Phys Fitness 39: 202-206. [Crossref]

27. Battie K, Kenny IC, Lyons M, et al. (2014) The effect of strength training on performance in endurance athletes. Sports Med 44: 845-865. [Crossref]

28. 28. Gobel FL, Nordstrom LA, Nelson RR, et al. (1978) The rate-pressure product as an index of myocardial oxygen consumption during exercise in patients with Angina Pectoris. Circulation 57: 549-556. [Crossref]
29. Carrick-Ranson G, Hastings JL, Bhella PS, Fujimoto N, Shibata S, et al. (2014) The effect of lifelong exercise dose on cardiovascular function during exercise. $J \mathrm{Appl}$ Physiol (1985) 116: 736-745. [Crossref]

30. Brandao MU, Wajngarten M, Rondon E, Giorgi MC, Hitonaka F, et al. (1993) Lef ventricular function during dynamic exercise in untrained and moderately trained subjects. J Appl Physiol 75: 1989-1995. [Crossref]

31. Fu T, Wang C, Lin P, Hsu C, Chern W, et al. (2013) Aerobic interval training improves oxygen uptake efficiency by enhancing cerebral and muscular hemodynamics in patients with heart failure. Int $J$ Cardiol 167: 41-50. [Crossref]

32. De Meersman RE (1993) Heart rate variability and aerobic fitness. Am Heart $J 125$ 726-731. [Crossref]

33. Akkerman M, van Brussel M, Hulzebos E, Vanhees L, Helders PJ, et al. (2010) The oxygen uptake efficiency slope: what do we know? J Cardiopulm Rehabil Prev 30: 357-373. [Crossref]

34. Buys R, Coeckelberghs E, Vanhees L, Cornelissen VA (2015) The oxygen uptake efficiency slope in 1411 Caucasian healthy men and women aged 20-60 years: reference values. Eur J Prev Cardiol 22: 356-363. [Crossref]

35. Scott CB, Littlefield ND, Chason JD, Bunker MP, Asselin EM (2006) Differences in oxygen uptake but equivalent energy expenditure between a brief bout of cycling and running. Nutr Metab (Lond) 3: 1. [Crossref]

Copyright: (C2017 Pasqua LA. This is an open-access article distributed under the terms of the Creative Commons Attribution License, which permits unrestricted use, distribution, and reproduction in any medium, provided the original author and source are credited. 